\title{
Fuzzy Chaos Whale Optimization and BAT Integrated Algorithm for Parameter Estimation in Sewage Treatment
}

\author{
Dr. Akey Sungheetha
}

Data Science SIG member,

Computer Science and Engineering,

School of Electrical Engineering and Computing,

Adama Science and Technology University,

Adama, Nazret, Ethiopia.

\section{Dr. Rajesh Sharma R}

Image Processing SIG member,

Computer Science and Engineering,

School of Electrical Engineering and Computing,

Adama Science and Technology University,

Adama, Nazret, Ethiopia.

\begin{abstract}
Biological and social issues rise with faults that occur in waste water treatment plant (WWTP). Nature as well as humans are negatively impacted by the dangerous effects of poorly treated wastewater. This paper combines the fuzzy logic, chaos theory, whale optimization algorithm (WOA) and BAT algorithm (FCW-BAT) to create a novel model for parameter estimation. The WWTP applications are exposed to FCW-BAT algorithm for identifying nonwell-structured domain, validating decision rules, cost reduction and estimation of several relevant attributes from the complete dataset. The significant data is retained while reducing the complete feature set using FCW-BAT prior to the classification process. Estimation of data uncertainty and fuzzification is performed with the cost function fast fuzzy c-means. The WOA parameters are estimated and tuned with the help of several chaos sequence maps. Complex real-time datasets consisting of missing values and several uncertainty features are tested and experimented. Shorter execution time, higher convergence speed, lower error and improved performance are obtained with the sine chaos map embedded in the proposed algorithm. Additionally, the WWTP sensor process faults may also be detected by the proposed model with great levels of accuracy enabling the system operators to make appropriate control decisions.
\end{abstract}


Journal of Soft Computing Paradigm (JSCP) (2021)

Vol.03/ No.01

Pages: $10-18$

http://irojournals.com/jscp/

DOI: https://doi.org/10.36548/jscp.2021.1.002

Keywords: Fuzzy c-means algorithm, Chaos theory, BAT algorithm, Waste water treatment plant, Whale optimization algorithm, Fault detection

\section{Introduction}

In order to remove contaminants from industrial and household sewage, an ensemble of process termed as waste water treatment is carried out. The inorganic as well as natural pollutants in the water makes it inappropriate for usage [1]. The biological and chemical wastes may be removed from the water used in everyday human life, industry as well as agriculture by means of waste water treatment plant (WWTP). Water line and sludge line are the two independent subsystems available in waste water treatment. The water line subsystem is the area of focus in this paper [2]. The measurements recorded by sensors are not always reliable in any automated system. For this purpose, detection and control of abnormal measurements on time is essential. A biological and global health hard problem may be caused by fault in WWTP [3]. Dangerous effects may be caused for both nature as well as human beings due to poorly treated waste water from the plant. Maximizing performance while minimizing defects in the treatment process is essential for efficient fault detection and management of waste water treatment plant. Several application areas are implemented with machine learning (ML) in a successful manner [4]. Knowledge acquisition problem is emphasized in several industrial plants for addressing reliable fault monitoring and detection as well as other complex application problems. Large number of features are presented by these issues, of which, not all are required for classification or clustering task. It is not possible to neglect the unavailable or unknown values [5].

It is highly desirable to enable the classification rules to be generated for the applications. For complex datasets, during the prediction or classification phase, it is challenging to find the most relevant features and enable feature selection [6]. It is a pressing matter to discover largescale data with hidden valuable knowledge that is available in it and extraction of the significant information. Feature selection may be performed using two major schemes namely wrapper approach and the filter approach. Fisher score, information gain, relief and mutual information as well as other ML techniques are insignificant in the filter approach. The data dependent criterion is optimized using a subset of features in this technique. The evaluation function consists of ML techniques in wrapper approach [7]. When compared to the filter approach, the wrapper approach offers better results. Sequential forward floating search, sequential random 
Journal of Soft Computing Paradigm (JSCP) (2021)

Vol.03/ No.01

Pages: $10-18$

http://irojournals.com/jscp/

DOI: https://doi.org/10.36548/jscp.2021.1.002

search, sequential backward floating search, sequential backward search, sequential forward search and other wrapper approaches are available that fall under the local minima and are computationally expensive [8]. Global optimization technique is essential for further improvement of the feature selection effect.

\section{Related Works}

Several issues in uncertainty, missing values, noise sensitivity, non-convergence to optimal solution, and time computation are faced by the classical ML algorithms working on WWTP [9]. Bio-inspired swarm intelligence algorithms like BAT algorithm and whale optimization algorithm (WOA) are integrated to provide a smart approach to deal with these issues. The WOA is a meta-heuristic swarm intelligence optimization scheme, proposed in the year 2016, that is based on modeling, imitation and observation of the humpback whales and their special hunting scheme [10]. The bubble-net feeding technique is the foraging scheme observed here. The WOA is used for solving several optimization problems in the engineering domains [11]. Intensification or exploitation and diversification or exploration are the two major factors balanced by the global optimizer using WOA. The current best solutions are searched in an intensive manner under exploitation and the best candidate is selected. The search space is randomly explored for new regions under the exploration process [12]. The WOA's stochastic nature has led to the lack of a clear boundary between the exploration and exploitation phases in a complex and nonlinear system [13].

Chaos theory may be used for balancing between diversification as well as the intensification rates while improving the local minima avoidance and convergence speed of WOA thereby improving its overall performance [8]. Prediction or control of nonlinear problems in dynamic systems which is otherwise challenging is performed using the chaos theory. The inner stochasticity performance and certainty are dealt by the chaos theory. Various chaos maps namely tent, sinusoidal, singer, sine, piecewise, logistic, iterative, Gauss, circle and Chebyshev are introduced and analyzed [5]. Various engineering applications are exposed to the model. Fuzzy rule-based systems and fuzzy sets have emerged largely during the last few decades and are widely accepted to represent and capture the smart systems framework as a dominant mechanism. Data uncertainty and ambiguity issues are addressed by the fuzzy sets [14]. Universal approximations are performed efficiently with appropriate fuzzy rules. The performance of fuzzy rules can be improved in various fields by integrating with swarm 
Journal of Soft Computing Paradigm (JSCP) (2021)

Vol.03/ No.01

Pages: $10-18$

http://irojournals.com/jscp/

DOI: https://doi.org/10.36548/jscp.2021.1.002

intelligence. Various engineering applications are exposed to the fuzzy swarm intelligence algorithms.

\section{Proposed Work}

Activate sludge system (ASS) are biological treatment schemes for waste water that is carried out in the WWTP process. With respect to a wide range of conditions, the operation of ASS can be adjusted due to its highly controllable property. Sludge line and water line are the two processes involved in the ASS. The proposed WWTP process and its steps are designed in a detailed manner. Primary, secondary and tertiary treatment processes are the constituents of the waterline. Solids are checked from the water at the primary treatment and decantation of the organic settleable matter is performed. The biological process is carried out during the secondary treatment where the water dissolves organic matter that is degraded by the multispecifically microbiological activity. During the tertiary treatment process, the effluent is created by separating the mixed liquor suspended solids (MLSS). Model simplicity, sensor faults and other reliable instrumentation lack leads to complex problems in the WWTP control process. Monitoring the sensor based faults and challenges in controlling the process are the major issues. An experienced and qualified personnel is required 24 hours a day during the plant operation which is a complex process. A lot of unmanageable variables are introduced to the plant like calculation of microorganisms and organic matter essential for preserving the water bodies and their ecological equilibrium. These phenomenon contribute to the social importance of these plants. Every day, a set of 45 features constituting the system variables are introduced and the behavior of the plant dataset is observed over a duration of 365 days.

The complex environmental process carried out by waste water treatment plants are challenging to manage and control. This paper combines the fuzzy logic, chaos theory, whale optimization algorithm (WOA) and BAT algorithm (FCW-BAT) to create a novel model for parameter estimation. In high dimensionality non-linear data, the issue of feature selection may be overcome using the proposed FCW-BAT algorithm. Often, multiple local optima are obtained by the existing algorithms used in waste water treatment due to its nonlinearity. A global meta-heuristic optimization algorithm is introduced to overcome this problem. The most recent global optimization algorithm introduced is the stochastic WOA. Despite the random distribution behavior, balancing between exploitation and exploration is the major challenging task in WOA. There is a unclear boundary between exploitation and exploration in complex 
Journal of Soft Computing Paradigm (JSCP) (2021)

Vol.03/ No.01

Pages: $10-18$

http://irojournals.com/jscp/

DOI: https://doi.org/10.36548/jscp.2021.1.002

and non-linear systems. There is no guarantee for a global solution as WOA experiences fall in local minima and low convergence due to the aforementioned reasons. Rather than studying the random behavior, the position of WOA is updated using several chaotic sequence maps. The convergence rate and optimal solution are affected by updating the position of WOA. These maps are also mathematically modeled. In order the guarantee the selected features in terms of goodness, determine the data uncertainty, decrease the local minima trapping chances and increase the convergence performance, the FCW is used as a cost function. Figure 1 represents the schematic diagram of the proposed FCW-BAT model.

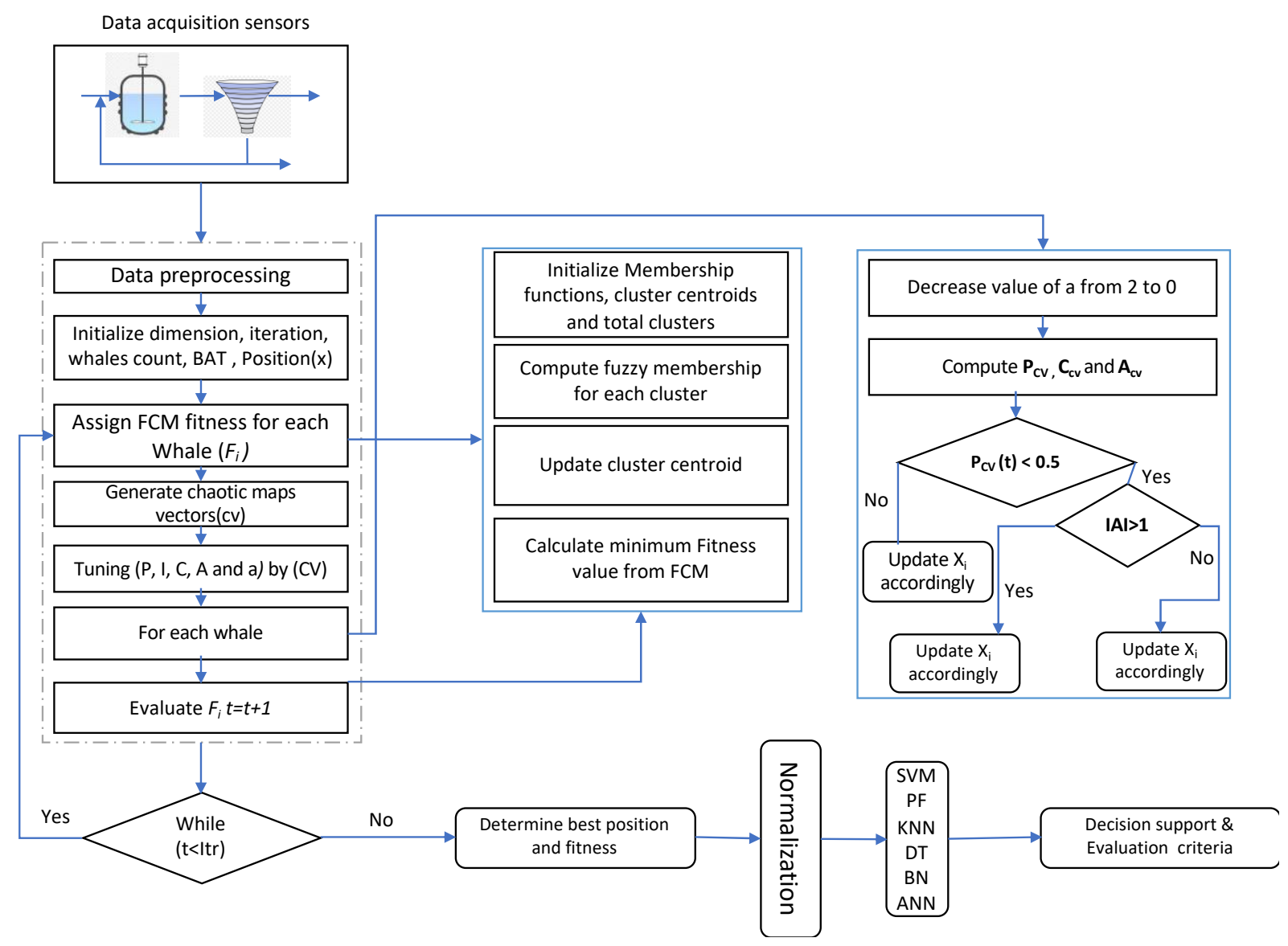

Fig. 1 Proposed FCW-BAT optimization model

\section{Results and Discussion}

An urban waste water treatment plant is observed and its sensor measures are obtained on a daily basis for creation of the WWTP dataset. For one complete year, the day-to-day water testing quantity, recordings of particles, chemical substances, wastage and so on are contained in the dataset. The plant circulates water for over 10,000 houses in the location with a flow of 
Journal of Soft Computing Paradigm (JSCP) (2021)

Vol.03/ No.01

Pages: 10-18

http://irojournals.com/jscp/

DOI: https://doi.org/10.36548/jscp.2021.1.002

$20,000 \mathrm{~m}^{3} /$ day. Along with this, the plant also receives waste water from industries. The WWTP variables and their distribution during the treatment process are analyzed in an extensive manner. Prediction of the plant operation state while predicting the treatment process faults is the major objective of this work. This domain has been regarded as ill-structured. Integer and real attributes are available in the dataset contributing towards its multivariate traits. It also consists of unknown information and missing values. The dataset is obtained from the UCI repository.

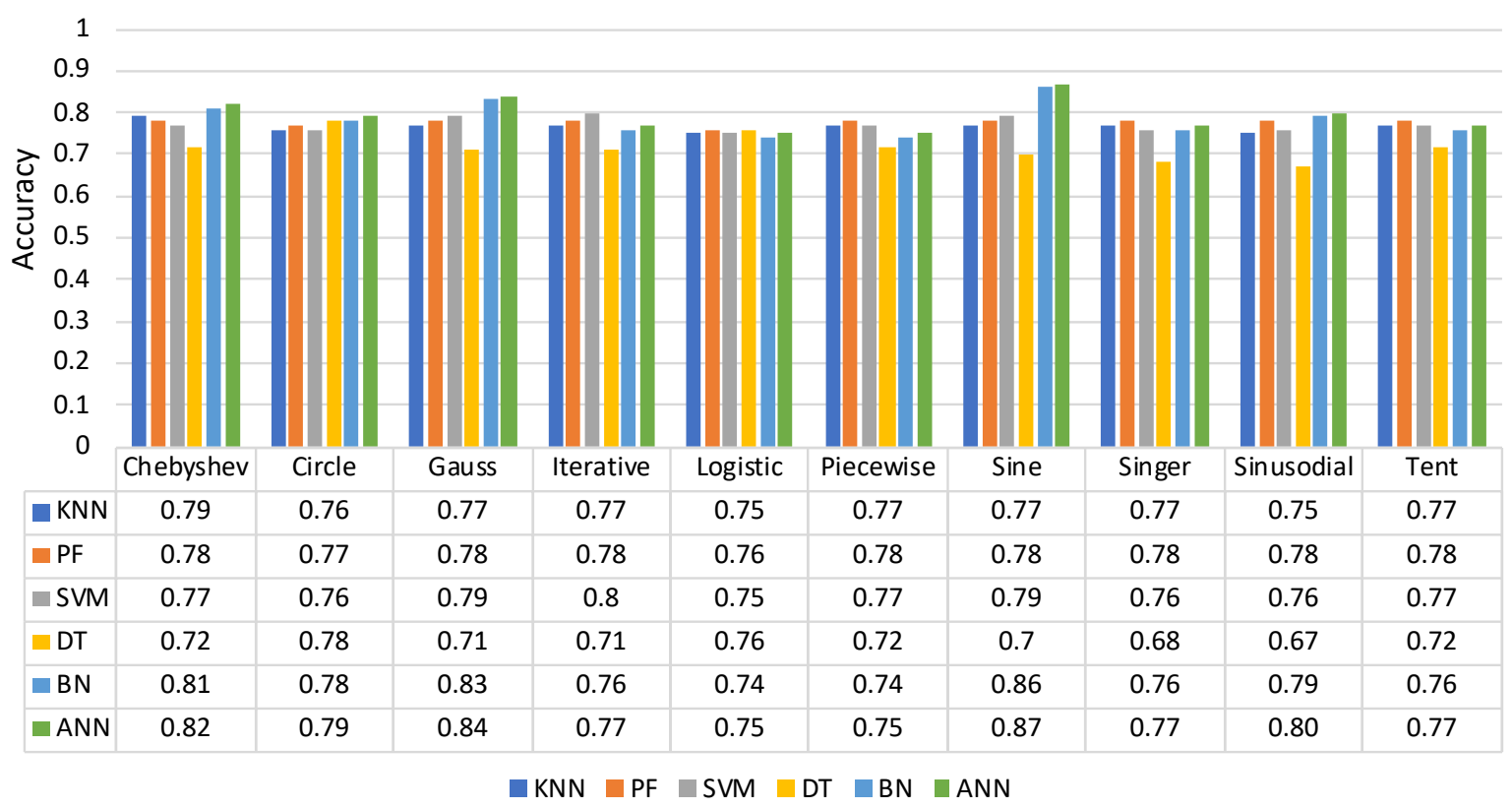

Fig. 2 Feature selection based classifier comparison

The proposed FCW-BAT algorithm offers highest accuracy of classification when compared to the existing automatic feature selection based classifiers. The NB classifier and sine chaos map as well as the Gauss map express the same accuracy of classification. Figure 2 provides the comparison of the classifier accuracy based on feature selection. Figure 3 represents the convergence of the proposed FCW-BAT algorithm with respect to the best scores. The local minima fall is avoided and the WOA is assisted while the algorithm convergence speed responds positively with the increase in iterations. Lesser CP average time or computation time (in seconds) is obtained by carrying out the optimal results. Quicker convergence speed and strong robustness is exhibited by the proposed FCW-BAT algorithm as seen in the plots. The algorithm is appropriate for real-time fault detection and requires less than 10 iterations to find the optimal solution. 
Journal of Soft Computing Paradigm (JSCP) (2021)

Vol.03/ No.01

Pages: $10-18$

http://irojournals.com/jscp/

DOI: https://doi.org/10.36548/jscp.2021.1.002

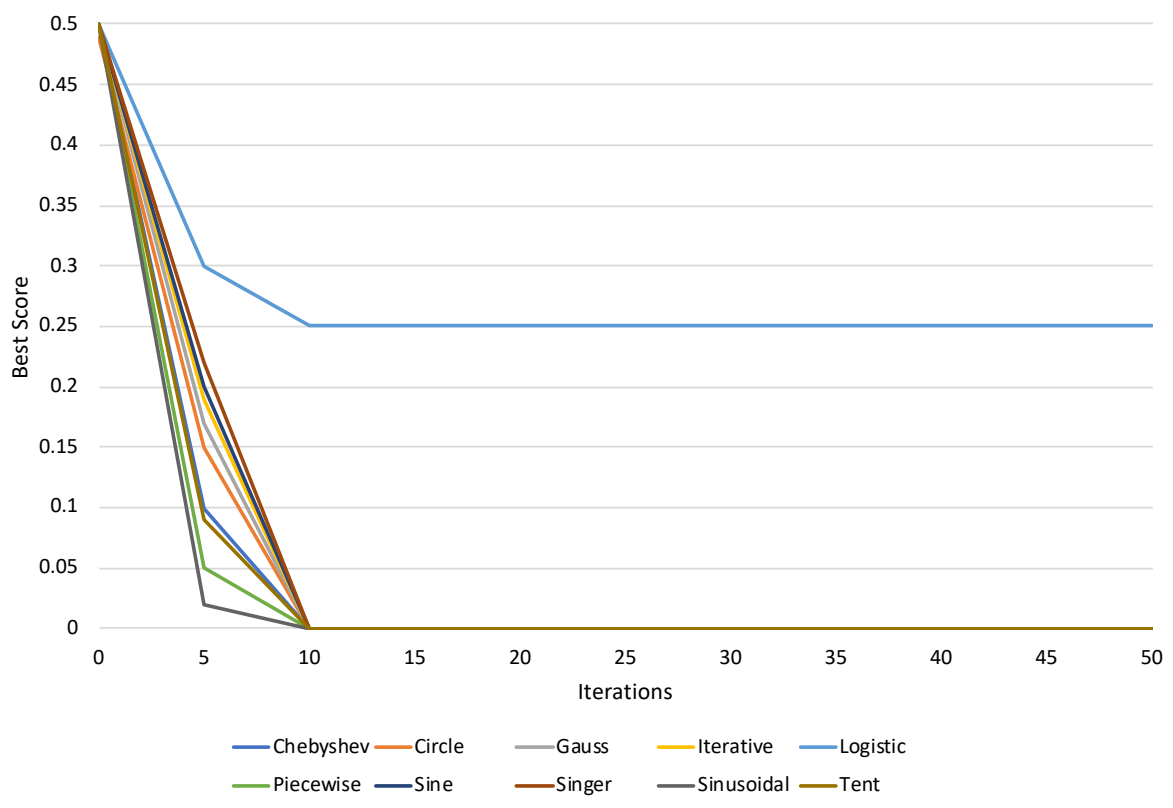

Fig. 3 Convergence of the proposed FCW-BAT approach

When compared to the existing algorithms, the accuracy of the proposed FCW-BAT algorithm is presented in figure 4 . The algorithm obtains a highest of $87 \%$ which is reasonably higher when compared to the overall performance of GWO, WOA, BAT, ALO, CALO, and BCCSA algorithms.

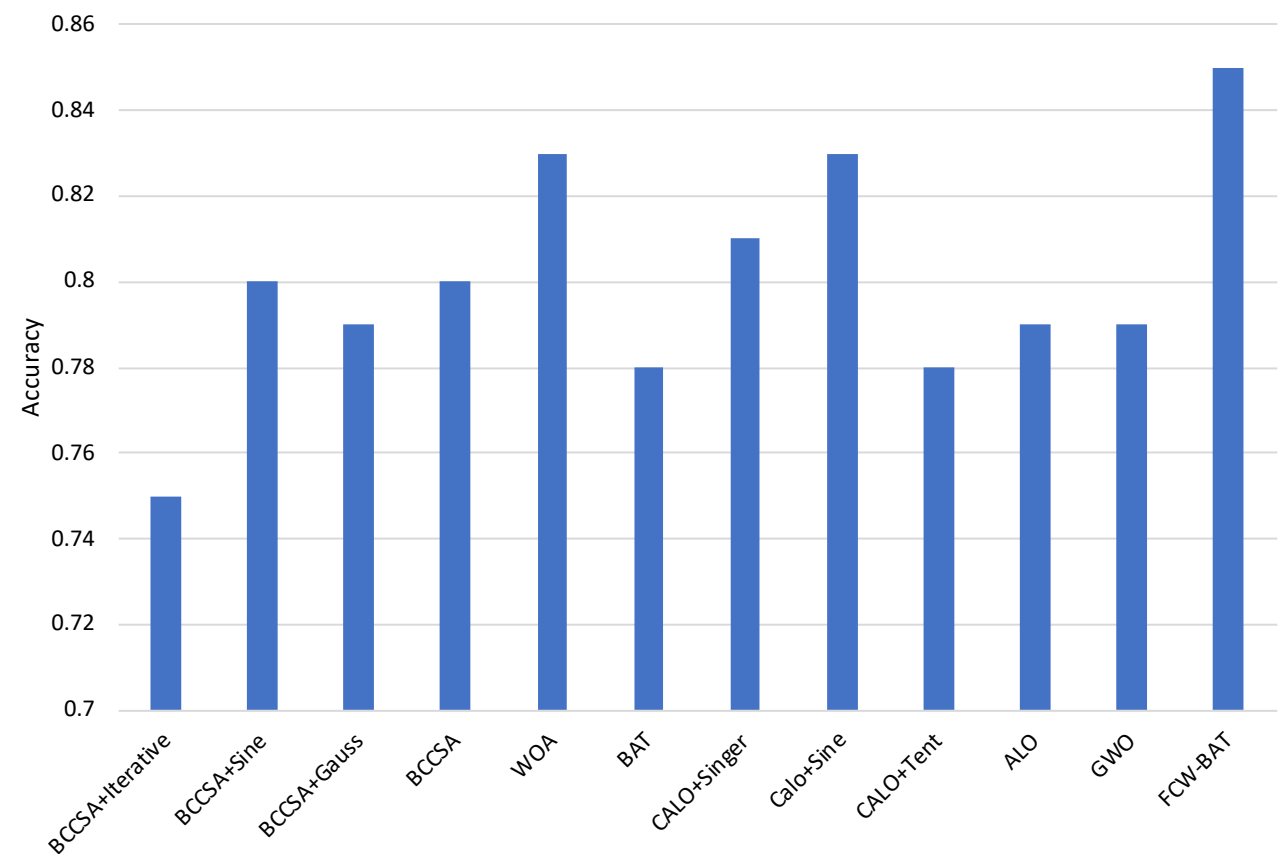

Fig. 4 Accuracy comparison between different feature selection optimization algorithms

ISSN: 2582-2640 (online) 
Journal of Soft Computing Paradigm (JSCP) (2021)

Vol.03/ No.01

Pages: $10-18$

http://irojournals.com/jscp/

DOI: $\underline{\text { https://doi.org/10.36548/jscp.2021.1.002 }}$

\section{Conclusion}

The field of intelligent systems is largely benefited with the development of a tool for detection, diagnosis as well as control of faults in a waste water treatment plant. A hybrid algorithm obtained on combining the fuzzy logic, chaos theory, WOA and BAT algorithm (FCW-BAT) is proposed. The agents in search space are evaluated while measuring the data uncertainty as well as fuzzification with a cost function provided by the fast fuzzy c-means. The convergence speed and performance of the dynamic process are enhanced while tuning the WOA parameters by merging swarm algorithm over several chaotic maps. Feature selection issue, one of the major problems of WWTP is addressed by the FCW-BAT algorithm. Large number of features, missing values, complex datasets with uncertainties and nonlinear datasets are tested with the proposed model and the model is validated. The proposed model is evaluated by adopting various statistical significant measures. Various recent and popular bio-inspired algorithms like GWO, WOA, BAT, ALO, CALO, BCCSA and so on are also compared with the proposed model. At specific AC values, the proposed model achieves highest accuracy with least optimization time consumption. The proposed FCW-BAT algorithm outperforms other existing algorithms discussed in the literature in terms of mean and best fitness value, RMSE, accuracy and time consumption. High accuracy and stability is also achieved by the proposed algorithm. Future work is directed towards ensuring stability of the proposed model while applying it to real-word engineering challenges. Further, integration of more features in the algorithm may be explored to enhance the reliability.

\section{References}

[1] Anter, A. M., \& Zhang, Z. (2019, October). Adaptive Neuro-fuzzy inference systembased chaotic swarm intelligence hybrid model for recognition of mild cognitive impairment from resting-state fMRI. In International Workshop on PRedictive Intelligence In MEdicine (pp. 23-33). Springer, Cham.

[2] Anter, A. M., Bhattacharyya, S., \& Zhang, Z. (2020). Multi-stage fuzzy swarm intelligence for automatic hepatic lesion segmentation from CT scans. Applied Soft Computing, 96, 106677.

[3] Abualigah, L., Shehab, M., Alshinwan, M., \& Alabool, H. (2019). Salp swarm algorithm: a comprehensive survey. Neural Computing and Applications, 1-21. 
Journal of Soft Computing Paradigm (JSCP) (2021)

Vol.03/ No.01

Pages: $10-18$

http://irojournals.com/jscp/

DOI: $\underline{\text { https://doi.org/10.36548/jscp.2021.1.002 }}$

[4] Houssein, E. H. (2019). Machine Learning and Meta-heuristic Algorithms for Renewable Energy: A Systematic Review. Advanced Control and Optimization Paradigms for Wind Energy Systems, 165-187.

[5] Ehteram, M., Ahmed, A. N., Ling, L., Fai, C. M., Latif, S. D., Afan, H. A., ... \& ElShafie, A. (2020). Pipeline Scour Rates Prediction-Based Model Utilizing a Multilayer Perceptron-Colliding Body Algorithm. Water, 12(3), 902.

[6] Seifi, A., Ehteram, M., \& Soroush, F. (2020). Uncertainties of instantaneous influent flow predictions by intelligence models hybridized with multi-objective shark smell optimization algorithm. Journal of Hydrology, 587, 124977.

[7] Karegowda, A. G., \& Devika, G. (2021). Meta-Heuristic Parameter Optimization for ANN and Real-Time Applications of ANN. In Applications of Artificial Neural Networks for Nonlinear Data (pp. 227-269). IGI Global.

[8] Mahmoud, K., Abdel-Nasser, M., Mustafa, E., \& M Ali, Z. (2020). Improved SalpSwarm Optimizer and Accurate Forecasting Model for Dynamic Economic Dispatch in Sustainable Power Systems. Sustainability, 12(2), 576.

[9] Giffler, B., \& Thompson, G. L. (1960). Algorithms for solving production-scheduling problems. Operations research, 8(4), 487-503.

[10] Srinivas, N., \& Deb, K. (1994). Muiltiobjective optimization using nondominated sorting in genetic algorithms. Evolutionary computation, 2(3), 221-248.

[11] Dutta, S., \& Banerjee, A. (2020). Highly Precise Modified Blue Whale Method Framed by Blending Bat and Local Search Algorithm for the Optimality of Image Fusion Algorithm. Journal of Soft Computing Paradigm (JSCP), 2(04), 195-208.

[12] Kumar, A. D. Flawless Attuning for Parameters of Power System Modulator Applying Grey Wolf Optimization.

[13] Karunakaran, V. (2019). a stochastic development of cloud computing based task scheduling ALGORITHM. Journal of Soft Computing Paradigm (JSCP), 1(01), 41-48.

[14] Bashar, A. (2019). Secure And Cost Efficient Implementation Of The Mobile Computing Using Offloading Technique. Journal of Information Technology, 1(01), 48-57. 